\title{
Atuação de Psicólogos em Alegações de Violência Sexual: Boas Práticas nas Entrevistas de Crianças e Adolescentes
}

\author{
Carlos Aznar-Blefari ${ }^{1}$ \\ Luiziana Souto Schaefer ${ }^{2}$ \\ Cátula da Luz Pelisoli ${ }^{3}$ \\ Luisa Fernanda Habigzang ${ }^{1}$ \\ ${ }^{1}$ Pontifícia Universidade Católica do Rio Grande do Sul, Porto Alegre, Rio Grande do Sul, Brasil \\ ${ }^{2}$ Instituto-Geral de Perícias do Rio Grande do Sul, Departamento Médico-Legal de Porto Alegre, Porto Alegre, Rio Grande do Sul, Brasil \\ ${ }^{3}$ Tribunal de Justiça do Rio Grande do Sul, Comarca de Passo Fundo, Brasil
}

\begin{abstract}
Resumo
Discussões sobre o papel de profissionais da Psicologia na escuta de alegações de violência sexual contra crianças e adolescentes têm emergido em todo país. Escuta especializada, depoimento especial e perícia psicológica são procedimentos previstos na legislação brasileira em diferentes momentos de uma alegação de violência sexual, dentro do Sistema de Garantia de Direitos. Enquanto os dois primeiros podem contar com profissionais de outras áreas, a perícia psicológica é atribuição privativa dos psicólogos. Tendo em vista que a principal fonte de informações sobre os eventos alegados é a criança, este artigo de revisão narrativa tem como objetivo discutir a escuta do psicólogo/a sobre alegações de violência sexual nos contextos da escuta especializada, do depoimento especial e da perícia psicológica. O artigo também tem como objetivo apresentar diretrizes gerais para entrevistas com crianças e adolescentes, consideradas na literatura como boas práticas nesse campo de atuação. Considerando a entrevista como o ponto comum entre esses três procedimentos, recomenda-se o uso de questões abertas, preparação do local em que a entrevista será conduzida e o uso de protocolos empiricamente validados para obtenção do relato sobre o evento alegado. Observou-se que tanto na literatura especializada como na legislação brasileira ainda se faz necessário esclarecer a operacionalização de "escuta especializada", pois pode dificultar a atuação efetiva dos profissionais que atuam em serviços de proteção e atendimento a crianças e adolescentes.

Palavras-chave: entrevista, prova pericial, abuso sexual na infância
\end{abstract}

Performance of psychologists in allegations of sexual violence: Good practices in Child and Adolescent Interviews

\begin{abstract}
Discussions about the role of psychologists in listening to allegations of sexual violence against children and adolescents have emerged across the country. Specialized listening, special testimony, and an expert legal psychological report are procedures provided for in Brazilian law at different steps of an allegation of sexual violence within the System of Guarantee of Rights. While the first 2 can rely on professionals from other fields, the expert legal psychological report is the private responsibility of psychologists. Given that the main source of information about the alleged events is the child, this narrative review article aims to discuss the psychologist's listening to allegations of sexual violence in the contexts of specialized listening, special testimony, and expert legal psychological report. The article also aims to present general guidelines for interviews with children and adolescents, considered in the literature as good practices in this field. Considering the interview as the common point between these 3 procedures, it is recommended the use of open questions, preparation of the place where the interview will be conducted, and the use of empirically validated protocols to obtain the report of the alleged event. It was observed that in the specialized literature as well as in the Brazilian legislation, it is still necessary to elucidate the operationalization of 'Specialized Listening', as it can hinder the effective performance of professionals working in protection and care services for children and adolescents. Keywords: Interview; Expert testimony; Child sexual abuse.
\end{abstract}

\section{Desempeño de psicólogos en denuncias de violencia sexual: Buenas prácticas en entrevistas a niños y adolescentes}

\section{Resumen}

Las discusiones sobre el papel de los profesionales de la psicología en la escucha de las denuncias de violencia sexual contra niños y jóvenes han surgido en todo el país. La escucha especializada, el testimonio especial y la pericia psicológica son procedimientos previstos en la legislación brasileña en momentos distintos de una denuncia de violencia sexual dentro del Sistema de Garantía de Derechos. Si bien los dos primeros pueden confiar en profesionales de otros campos, la evaluación psicológica (pericia psicológica) es la tarea privada del psicólogo. Dado que la principal fuente de información sobre los supuestos eventos es el niño, este artículo de revisión narrativa tiene como objetivo discutir la escucha del psicólogo en las denuncias de violencia sexual en los contextos especializados, testimonio especial y evaluación psicológica (pericia psicológica). El artículo también tiene como objetivo presentar directrices generales para entrevistas con niños y jóvenes, consideradas en la literatura como buenas prácticas en este campo. Considerando la entrevista como el punto común entre estos tres procedimientos, se recomienda el uso de preguntas abiertas, la preparación del lugar donde se realizará la entrevista y el uso de protocolos empíricamente validados para obtener el evento informado. Se observó que, en la literatura especializada, así como en la legislación brasileña, aún es necesario aclarar la operacionalización de la "Escucha Especializada", ya que puede obstaculizar el desempeño efectivo de los profesionales que trabajan en los servicios de protección y cuidado para niños y jóvenes. Palabras clave: Entrevista; Testimonio de experto; Abuso sexual infantil. 


\section{Introdução}

As situações de suspeita de violência sexual contra crianças e adolescentes adentram ao Sistema de Garantia de Direitos com algumas especificidades. Por considerar que a vítima ou testemunha é um sujeito em condição peculiar de desenvolvimento e objeto de proteção integral na legislação brasileira (Brasil, 1990), deve haver uma preocupação sobre como os procedimentos são realizados em todos os momentos, desde antes do inquérito policial até o processo judicial. Essa preocupação é ainda maior por conta da natureza da violência alegada, que tem suas particularidades. A violência sexual envolve, muitas vezes, agressores intrafamiliares, a presença de ameaças, sentimentos de culpa, medo, vergonha e demais consequências para a vítima, além de possíveis alterações na configuração familiar e outras mudanças de vida decorrentes da revelação da situação de violência (Habigzang, Ramos, \& Koller, 2011).

A Lei 13.431 de 4 de abril de 2017 inovou ao tornar o depoimento especial o procedimento padrão de oitiva de crianças e adolescentes no Sistema de Justiça e ao diferenciá-lo da Escuta Especializada, que ocorre em outros órgãos da rede de atendimento, sem objetivo de produção de prova (Brasil, 2017; 2018). A lei estabelece o sistema de garantia de direitos da criança e do adolescente vítima ou testemunha de violência, cria mecanismos de prevenção à violência e estabelece medidas de assistência e proteção e, além disso, indica a necessidade de capacitação profissional continuada e preferencialmente multidisciplinar para a utilização de técnicas de entrevista cientificamente reconhecidas. Por fim, a nova doutrina estabelece questões relativas à integração das políticas de atendimento nas áreas da saúde, assistência social, segurança pública e justiça, que envolvem desde os encaminhamentos das notificações recebidas até a possibilidade de criação de serviços específicos para esse público, medidas de proteção em procedimentos investigativos, entre outros. Especificamente, a lei não estabelece quais profissionais devem realizar a Escuta Especializada - EE ou o Depoimento Especial - DE.

A nova lei, no entanto, não exclui tampouco diminui a importância da perícia psicológica, que continua a ocorrer com base em princípios legais tanto na fase de inquérito policial, quando é demandada pela autoridade policial (Delegado de Polícia), quanto na fase processual, quando é requerida pelo magistrado ou promotor de Justiça. A previsão legal encontra-se no Código de Processo Penal (Brasil, 1941), que aborda outros tipos de prova pericial, não exclusivamente psicológica. Diferentemente da avaliação que acontece no contexto da Psicologia Clínica, a avaliação pericial visa à obtenção de informações que subsidiem uma tomada de decisão (Rovinski \& Pelisoli, 2019) e, assim como toda avaliação psicológica, é um atributo exclusivo do profissional psicólogo/a (CFP, 2018).

$\mathrm{O}$ ponto comum entre os três procedimentos - EE, DE e perícia psicológica é a realização de entrevistas com as crianças/adolescentes. Entretanto, o estabelecimento de programas de capacitação profissional continuada (conforme estabelecido pela Lei 13.431/17) para a realização de entrevistas é realidade em apenas alguns municípios e, até então, esforços em mensurar os efeitos de tais capacitações são isolados (e.g., Aznar-Blefari \& Padilha, 2015; Hackbarth, Williams, \& Lopes, 2015). Ainda se observa o despreparo da rede de proteção em fornecer atendimentos adequados por lacunas na formação profissional (Conselho Federal de Psicologia [CFP], 2009). A capacitação profissional e a habilidade do/a entrevistador/a em conduzir a entrevista são fatores essenciais na obtenção de informações relevantes ao caso (Lamb, Hershkowitz, Orbach, \& Esplin, 2008). Uma entrevista conduzida de forma inadequada pode resultar na necessidade da repetição de procedimentos, aumentando assim o risco de violência institucional ou revitimização (conforme descrito pela Lei 13.431/17). Há também risco aumentado de sugestionabilidade e, consequentemente, descrença sobre as alegações da violência sexual infantil (Faller, 2015; Lamb et al., 2008).

Considerando esse contexto, este artigo tem como objetivo abordar e diferenciar a atuação do psicólogo/a em alegações de violência sexual contra crianças e adolescentes na escuta especializada, no depoimento especial e na perícia psicológica. Destacando-se a necessidade de capacitação continuada dos profissionais (Lei 13.431/17), o artigo também apresenta diretrizes gerais (i.e., boas-práticas) para a entrevista com crianças e adolescentes vítimas e testemunhas de violência.

\section{Escuta Especializada e Depoimento Especial}

Dentre outras questões, a Lei 13.431/17 define as diferentes formas de violência e estabelece que "a criança e o adolescente serão ouvidos sobre a situação de violência por meio de escuta especializada e depoimento especial" (Brasil, 2017). Contudo, observa-se tanto na Lei quanto no Decreto 9603/2018 (Brasil, 2018), o maior destaque para operacionalização do depoimento especial e poucas orientações sobre como 
a escuta especializada deve ser realizada. Ambos os procedimentos deverão ocorrer mediante alguns cuidados, como limitar o relato estritamente ao necessário para o cumprimento de sua finalidade, a necessidade de se resguardar a criança vítima ou testemunha de qualquer contato com o/a suposto/a agressor/a, entrevistá-la em local apropriado e acolhedor, com infraestrutura e espaço físico que garantam sua privacidade.

A Escuta Especializada - EE é definida como "o procedimento de entrevista sobre situação de violência com criança ou adolescente perante órgão da rede de proteção" (Brasil, 2017). Trata-se do momento em que a criança ou adolescente contará para alguém da rede de proteção sobre o que aconteceu com ela. A rede, ao tomar conhecimento sobre o ocorrido, deverá imediatamente comunicar as autoridades policiais (e.g., Ministério Público) sob risco de ferir os pressupostos da Lei 8.069 conhecida nacionalmente como Estatuto da Criança e do Adolescente (Brasil, 1990). O objetivo da escuta não é a produção de provas, mas sim a comunicação que viabilize a proteção integral da criança ou adolescente (Conselho Nacional do Ministério Público [CNMP], 2019).

A escuta especializada deve ter como objetivo obter informações "estritamente ao necessário para o cumprimento de sua finalidade" (Brasil, 2017) e assegurar o acompanhamento da vítima ou da testemunha para a superação da violação sofrida (Brasil, 2018). Deve ser uma escuta voltada ao acolhimento em que deve manter-se o cuidado de evitar possíveis intervenções sugestivas que possam interferir na contaminação da prova. A EE define-se muito mais como uma escuta que remete a procedimentos clínicos, de cuidado e proteção, em detrimento da preocupação em relação à produção de prova (Rovinski \& Pelisoli, 2019).

Por sua vez, o Depoimento Especial - DE, compreende a "oitiva de criança ou do adolescente vítima ou testemunha de violência perante autoridade policial ou judiciária” (Brasil, 2017). O depoimento especial deverá reger-se por protocolos, com prioridade para a realização em um único momento e em sede de antecipação de prova, evitando danos secundários (e.g., revitimização, contaminação/indução/sugestão). Por suas definições, fica claro que, enquanto o DE é realizado com o objetivo de produção de prova, a EE não tem essa proposta. Por esse motivo, talvez, é que as regulamentações sobre como ela deve ser realizada não são tão exigentes e não estão descritas com o mesmo grau de detalhamento que o DE, tanto na Lei 13431/2017 quanto no Decreto $9603 / 2018$.
No Brasil, as principais experiências de depoimento especial envolvem assistentes sociais e psicólogos, ainda que a legislação não defina de maneira determinista os profissionais que devem realizá-lo (Brasil, 2017, 2018). Os entrevistadores atuam por meio do sistema Closed Circuit Television - CCTV, que coleta depoimentos por meio de um circuito fechado de televisão e de videogravação e é utilizado pela maior parte dos países (64\%) (Santos \& Gonçalves, 2009). Assim, na medida em que as questões legais estão definidas, percebe-se necessidade de discussões mais concretas a respeito de questões de ordem técnica, ou seja, a forma de proceder junto à criança ou adolescente vítima no momento da entrevista propriamente dita.

Além de questões procedimentais, como a gravação do depoimento especial em áudio e vídeo e a transmissão da entrevista em tempo real para a sala de audiências, a lei busca assegurar à criança ou ao adolescente a livre narrativa sobre a situação vivida. Ao profissional que realiza a entrevista é garantida a intervenção sempre que considerar necessária, com técnicas que permitam a elucidação dos fatos, bem como the é garantido que adapte as perguntas feitas pela sala de audiências à linguagem de melhor compreensão da criança ou adolescente.

Um estudo empírico envolvendo 20 trabalhadores do Sistema de Justiça incluiu operadores do Direito e psicólogas com experiência em depoimento especial de crianças e adolescentes (Pelisoli \& Dell'Aglio, 2016). Além de verificar que o depoimento especial buscava tanto a comprovação dos fatos alegados quanto a proteção da vítima, as autoras puderam concluir que o treinamento posterior recebido especificamente para a condução das entrevistas era mais importante do que a área de formação desses profissionais. Nesse sentido, ainda que alguns operadores do Direito indicavam certa predileção pelo profissional da Psicologia, os participantes, de um modo geral, percebiam que o curso superior em que o/a entrevistador/a se graduou não se mostrava tão importante quanto o conhecimento sobre técnicas de entrevista, teorias sobre o desenvolvimento humano e personalidade, além de habilidades de acolhimento, o que é inclusive destacado pelo CNJ em sua recomendação (CNJ, 2010).

É fundamental, no entanto, enfatizar que o depoimento especial pode ser conduzido tanto por psicólogos, como também por outros profissionais, desde que habilitados em entrevista forense com crianças. Entrevistar crianças, portanto, não é uma prática 
restrita da Psicologia. Diferentemente, a perícia psicológica só pode ser conduzida por profissional habilitado nessa profissão. A perícia envolve mais do que a entrevista forense com a criança, compondo-se a partir de um conjunto de técnicas e procedimentos (Schaefer, Rossetto, \& Kristensen, 2012).

\section{Aspectos Legais e Técnicos da Perícia Psicológica}

A violência sexual contra crianças e adolescentes é um crime tipificado em diversos capítulos do Código Penal Brasileiro (Brasil, 1940). Quaisquer suspeitas dessa natureza precisam ser notificadas e, posteriormente, investigadas pelas autoridades policiais que deverão reunir todos os elementos probatórios que irão compor o inquérito policial. $\mathrm{O}$ resultado da investigação da infração penal é enviado ao Ministério Público e, caso o inquérito policial tenha trazido indícios suficientes da autoria e do cometimento do crime, o Ministério Público oferecerá a denúncia que, posteriormente, irá instaurar o processo judicial (Brasil, 1941 - Arts. 100 a 106). Portanto, os exames periciais são importantes elementos probatórios e, por vezes, decisivos, para a instauração do processo judicial.

A prova pericial no âmbito criminal está prevista no Código de Processo Penal e constitui um importante elemento de prova. É dito, no artigo 158 do referido código, que "quando a infração deixar vestígios, será indispensável o exame de corpo de delito, direto ou indireto, não podendo supri-lo a confissão do acusado" (Brasil, 1941). Portanto, durante a fase de inquérito policial, a autoridade deverá solicitar todos os exames periciais indispensáveis para a elucidação dos fatos, entre os quais se inclui a perícia psicológica. É importante destacar que, tanto no Código de Processo Penal (Brasil, 1941) quanto na Lei Federal 12.030/2009 (Brasil, 2009), é referido que as perícias de natureza criminal são realizadas por peritos oficiais, portadores de diploma de curso superior, sendo exigido concurso público e formação acadêmica específica detalhada em regulamento, de acordo com a necessidade de cada órgão e por área de atuação profissional.

No contexto das alegações de violência sexual, os exames médico-legais e as perícias em saúde mental são elementos cruciais na investigação desses crimes. Devido a circunstâncias particulares do tipo de interação sexual entre uma criança e o/a agressor/a que está em um estágio de desenvolvimento psicossexual mais avançado, a maioria dos casos não deixa vestígios físicos ou biológicos (Jardim \& Magalhães, 2010). Além disso, pela própria dinâmica do abuso, a avaliação da criança torna-se um dos principais elementos de prova (Lamb, Brown, Hershkowitz, Orbach, \& Esplin, 2018).

As perícias em saúde mental são realizadas por psicólogos ou psiquiatras e, nos casos de avaliações de suspeitas de violência sexual contra crianças, tais perícias têm, entre seus objetivos, avaliar a capacidade geral da criança para testemunhar sobre uma experiência abusiva que ela possa ter vivenciado; avaliar o impacto que as possíveis experiências abusivas possam ter desencadeado na saúde mental e no funcionamento geral das vítimas (Schaefer et al., 2012); e discutir o nexo causal entre a hipótese da situação abusiva e o impacto na saúde das vítimas. Para isso, é necessário garantir que a informação fornecida pela criança tenha sido obtida a partir de técnicas e de protocolos de entrevista forense que tenham evidências científicas e que sejam destinados para esse propósito.

No que diz respeito à avaliação da capacidade de testemunhar da criança, observa-se que possíveis quadros sintomatológicos ou ainda déficits cognitivos podem dificultar o relato da vítima (Peixoto, 2011). Especificamente quanto à perícia psicológica, a função pericial é subsidiar as decisões judiciais e inquéritos policiais com consideração aos limites e às possibilidades que a ciência psicológica é capaz de contribuir. Portanto, na perícia psicológica em casos de suspeita de violência sexual contra crianças e adolescentes, é fundamental que o profissional, além de buscar obter o relato sobre possíveis situações de violação de direitos, realize o exame do estado mental do entrevistado, com destaque para a avaliação das capacidades cognitivas e de orientação, das condições de memória e dos recursos linguísticos da criança. Tais informações são essenciais para se constatar que a criança apresenta condições cognitivas mínimas para emitir um relato livre sobre acontecimentos por ela vivenciados e, consequentemente, sobre o suposto abuso (Peixoto, Ribeiro, \& Alberto, 2013).

$\mathrm{Na}$ avaliação das alegações de violência sexual, outro aspecto importante e bastante discutido é quanto à avaliação da sintomatologia da criança, tendo em vista que alterações comportamentais e psicológicas podem ter sido desencadeadas em virtude dessa ocorrência (Dannlowski et al., 2012; Habigzang et al., 2008; Kendall-Tackett, Williams, \& Finkelhor, 1993). $\mathrm{O}$ quadro sintomatológico associado à violência sexual é amplo, não havendo um único sintoma que caracterize exclusivamente as vítimas, assim como crianças podem ter vivenciado a violência e não apresentarem sintomas (Kendall-Tackett et al., 1993; Pears, 
Kim, \& Fish, 2008). Portanto, a avaliação do quadro sintomatológico não é suficiente para reforçar a hipótese de violência sexual diante da ausência do relato da criança sobre a situação (Schaefer, 2014). Ainda assim, no contexto forense, sugere-se que a avaliação da sintomatologia e do exame do estado da criança sejam realizados pelos profissionais da saúde mental como um complemento à entrevista para obtenção do relato do abuso. Tal procedimento poderá ser determinante para a identificação de situações de risco à integridade física e psíquica da vítima, além de fundamental para a determinação de medidas protetivas e futuros encaminhamentos (Schaefer, 2014).

Mensurar o impacto da violência sexual na saúde mental das vítimas pode também corroborar outras evidências, aumentando a credibilidade da avaliação forense (Everson \& Faller, 2012; Schaefer et al., 2012). Em suma, é importante ficar claro que a violência sexual é um evento traumático cuja natureza varia ao longo de um contínuo de gravidade e as experiências das crianças podem ser completamente diferentes, influenciando, consequentemente, o impacto do evento para cada uma delas (Whit-Wooslwy, 2019).

Portanto, avaliar indicadores psicológicos e comportamentais no contexto forense é importante não apenas para reforçar uma hipótese de violência sexual, mas para apontar aspectos que necessitam de maiores investigações ou para demonstrar o impacto de determinada ocorrência na saúde das vítimas, quando esse nexo causal for possível (Schaefer, Brunnet, Lobo, Carvalho, \& Kristensen, 2018). Em alguns casos, tais informações podem ser determinantes para o desfecho do processo judicial (Peixoto, 2011). Além disso, a avaliação do impacto psicológico, no âmbito da perícia oficial, também pode ser determinante nos casos em que as vítimas e seus familiares ajuízam uma ação indenizatória na esfera cível e o Estado e/ou o/a agressor/a se responsabilizam com o ressarcimento das custas de cuidados médicos e psicológicos e outros tipos de reparos que podem ser necessários em virtude da situação abusiva experienciada.

\section{Boas-Práticas em Entrevistas Forense}

Nos três procedimentos descritos, a saber: escuta especializada, depoimento especial e perícia psicológica, a entrevista é uma das técnicas privilegiadas de acesso às alegações de violência sexual contra crianças, especificamente por ser baseada em protocolos estruturados e testados empiricamente. Justifica-se a utilização de protocolos de entrevista forense porque há indicadores suficientes de que eles melhoram significativamente a qualidade e a quantidade de elementos trazidos no relato da criança (Lamb, 2015; Lamb et al., 2018).

Em razão da dinâmica da violência, da comum ausência de outras testemunhas e de vestígios físicos ou biológicos, o relato da vítima acaba sendo um dos principais elementos de prova (Herman, 2010; Jardim \& Magalhães, 2010). Entretanto, a obtenção de tal relato não é tarefa fácil, exigindo capacitação contínua dos entrevistadores (Lamb et al., 2018).

O profissional deve realizar uma entrevista que respeite a fase do desenvolvimento em que a criança se encontra (Lamb et al., 2008). Deve-se levar em consideração que o desenvolvimento infantil depende, em parte, da estimulação recebida do ambiente, principalmente dos cuidadores. Crianças que são estimuladas a relatar eventos passados tendem a ser mais informativas, apresentando uma memória autobiográfica mais desenvolvida (Salmon \& Reese, 2016).

A maior parte das crianças que foram expostas a eventos abusivos revelam o que aconteceu em uma entrevista forense, principalmente se anteriormente já houve uma revelação (Lamb et al., 2018). Contudo, algumas características do evento abusivo e da própria criança podem estar relacionadas à maior relutância à revelação. Tendem a ser mais complexas entrevistas com crianças em idade pré-escolar devido ao foco atencional mais limitado (Peixoto et al., 2013); crianças que sintam medo das consequências da revelação, vergonha, constrangimento e medo de que não acreditem em seu relato (Collin-Vézina, De La Sablonnière-Griffin, Palmer, \& Milne, 2015); casos em que o abuso foi intrafamiliar (Lamb et al., 2018); e ainda meninos tendem a ter mais dificuldade em relatar o evento abusivo (Hershkowitz, Lamb, Orbach, Katz, \& Horowitz, 2012; Hohendorff, Habigzang, \& Koller, 2014).

Dessa maneira, os profissionais, ao se depararem com entrevistas em que a criança demonstre uma postura mais relutante em revelar o evento abusivo, tendem a adotar uma postura de menos suporte (i.e., rapport) ou ainda a utilizarem técnicas secundárias, como brinquedos, bonecas anatômicas, desenhos etc. (e.g., Brown, 2011; Katz, Klages, \& Hamamma, 2018).

O estabelecimento de rapport é indicado como boa prática nos principais guias de entrevista investigativa (APSAC, 2012; Home Office, 2011). Entende-se por rapport uma etapa da entrevista destinada à construção de uma relação baseada em confiança mútua, respeito e aceitação por meio de uma conversação amigável, contato visual, sorrisos, braços descruzados, 
postura de observação e uso de enunciados abertos, solicitando descrições sobre si e estados emocionais (Saywitz, Larson, Hobbs, \& Wells, 2015). Mesmo se tratando de uma etapa da entrevista, é importante que o/a entrevistador/a mantenha tal postura no decorrer de toda a interação, sempre atento/a à estados emocionais da criança (Hershkowitz et al., 2017).

Por mais reconhecida que seja a importância do estabelecimento de rapport (Saywitz, et al., 2015), a literatura carece de evidências empíricas de como deve-se operacionalizar tal prática em ambiente forense. Uma revisão sistemática objetivou avaliar quais as evidências científicas que serviriam de suporte ao desenvolvimento de rapport em contexto forense, para tanto, estudos publicados entre 1990 e 2014 indexados nas bases PsycInfo, PubMed, Sociological Abstracts, Social Services Abstracts, Web of Knowledge e Cochrane Central foram analisados. De 2761 artigos avaliados, foram encontrados três estudos empíricos sobre o desenvolvimento de rapport em entrevistas investigativas com crianças e adolescentes entre quatro e 17 anos de idade. Os achados sugerem que o rapport deve ser realizado por meio de enunciados abertos e que tal técnica estaria relacionada a um número maior de detalhes na fase substantiva da entrevista (i.e., fase na qual se investiga o tema alegado) (Saywitz et al., 2015).

Recentemente, um estudo avaliou a efetividade do treinamento de entrevistadores para responderem sensivelmente a entrevistas com crianças relutantes, utilizando enunciados abertos de apoio/suporte ao questionar crianças. Para que tal análise fosse possível, os autores desenvolveram um método de codificação de aspectos de suporte adequado e inadequado no rapport. Por suporte adequado apresentaram seis categorias: (1) Falar com a criança chamando-a pelo nome; (2) Reforçar positivamente a cooperação da criança agradecendo ela por ouvir as questões ou por fornecer detalhes; (3) Iniciar o rapport expressando interesse em conhecer a criança ou sendo gentil; (4) Enfatizar o rapport expressando cuidado ou preocupação pela criança ou ainda se apresentando para a criança como sendo uma pessoa confiável; (5) Ser empático com a criança quando ela expressar emoções, aceitando, repetindo ou questionando sobre (deve-se evitar a sugestionabilidade quanto às emoções das crianças, uma criança chorando, não necessariamente está expressando tristeza); (6) Encorajar a criança a fazer uma revelação, enfatizando que ela é a única fonte de informações sobre o que aconteceu ou oferecendo apoio (Hershkowitz et al., 2017).
Por suporte inadequado durante o rapport, os autores descreveram cinco categorias: (1) Oferecer suporte de forma sugestiva, assumindo hipóteses sobre estados emocionais e afins (e.g., "Você não precisa ficar com medo" quando a criança não mencionou que está com medo); (2) Confrontações sobre o que foi mencionado pela criança (e.g., "Mas como isso pode ter acontecido"); (3) Causar algum tipo de desconforto por interromper a criança, criticando, coagindo ou errando o nome dela; (4) Fornecer garantias infundadas e fazer promessas que não podem efetivamente ocorrer, como, por exemplo, afirmando que nada vai acontecer com a criança ou o/a agressor/a; (5) Ignorar os pedidos da criança e questões quando o/a entrevistador/a não adotar uma postura de apoio e suporte (Hershkowitz et al., 2017).

Diferentemente do contexto clínico, em que a sala apropriada à psicoterapia do público infantil é composta por recursos lúdicos, como jogos, brinquedos, bonecos e material de desenho, não é indicado que a sala onde será conduzida a entrevista forense contenha esses elementos (Hill, 2017; Lamb et al., 2018; Poole \& Bruck, 2012). Uma das justificativas é a importância de que a criança se mantenha atenta e concentrada na tarefa narrativa (APSAC, 2012; Lamb et al., 2018; Peixoto et al., 2013).

Dentre os elementos secundários à entrevista forense, destacam-se bonecas anatômicas e o uso de desenhos. Bonecas anatômicas têm sido utilizadas para clarificar e elaborar relatos sobre toques abusivos. Tal prática pode aumentar o número de erros e diminuir a precisão do relato (Hill, 2017; Lamb et al., 2018). Em paralelo à utilização de entrevistas realizadas por meio de protocolos, o uso de bonecas anatômicas não melhora a quantidade de detalhes sobre os eventos alegados, mas sim há um aumento na quantidade de erros (Salmon, Pipe, Malloy, \& Mackay, 2011). Outro risco da utilização de bonecas anatômicas está relacionado à chance de a criança começar a brincar e não mais ser colaborativa em descrever os eventos de interesse (Thierry, Lamb, Orbach, \& Pipe, 2005).

Desenhos em entrevistas geralmente são utilizados como uma ferramenta de comunicação não verbal de fácil acesso com o objetivo de facilitar o estabelecimento de rapport ou ainda clarificar relatos. Contudo, estudos têm demonstrado que a utilização desses recursos pode aumentar o risco de relatos falsos quando profissionais utilizam questões sugestivas sobre o desenho (Hill, 2017). A literatura indica que não se deve analisar o conteúdo dos desenhos (Brown, 2011). 
Outro aspecto importante para a condução de uma entrevista forense seria o estabelecimento de regras iniciais de comunicação, tendo em vista que crianças tendem a se comportar de maneira a querer agradar adultos (Lamb et al., 2008). Dessa forma, crianças podem ser vulneráveis a responderem questões sugestivas de forma a buscar acertos (Volpini, Melis, Petralia, \& Rosenberg, 2016).

Ao iniciar a condução da entrevista, é essencial que o profissional se apresente para a criança e solicite autorização para que a entrevista seja gravada. Logo após, o profissional deve esclarecer as regras da entrevista, explicando à criança que ela será solicitada a contar detalhes sobre eventos que ela experienciou, que é necessário que ela relate somente a verdade e que não há problema caso ela não se lembre, não compreenda ou não saiba responder o que lhe está sendo perguntado (APSAC, 2012; Lamb et al., 2008; Lamb et al., 2018). O/A entrevistador/a também deve informar a criança que ela pode corrigi-lo, caso seja pertinente. Quando o/a entrevistador/a ressaltar a importância de que seja dito somente a verdade, é relevante avaliar se a criança tem a compreensão desse conceito e sabe distinguir a verdade da mentira (Lamb et al., 2008). Para isso, $\mathrm{o} / \mathrm{a}$ entrevistador/a poderá fazer perguntas para a criança sobre conteúdos neutros em que ambos saibam a resposta e verificar com a criança se aquela informação fornecida pelo/a entrevistador/a é verdadeira ou falsa (APSAC, 2012).

Outro consenso na literatura é quanto ao tipo de questões que o/a entrevistador/a deve utilizar para investigar os eventos alegados. De maneira geral, os enunciados formulados pelo/a entrevistador/a devem ampliar a capacidade narrativa e estimular a capacidade de evocação livre da memória dos entrevistados, ou seja, a aptidão deles em contar livremente o que lhes ocorreu da maneira mais próxima possível com a situação vivenciada e com o maior número de detalhes. O/A entrevistador/a também deve evitar o uso de enunciados sugestivos e minimizar outras possíveis interferências no relato dos entrevistados (Lamb et al., 2008; Lamb et al., 2018; Lyon, 2010; Peixoto et al., 2013).

Enunciados abertos possibilitam uma maior compreensão do que o/a entrevistador/a gostaria de saber sem sugerir novas informações. Ao se utilizar tal tipo de enunciado, há um aumento significativo no número de detalhes fornecidos pela criança (e.g., "Me conta tudo sobre isso") (Lamb et al., 2008; Lamb et al., 2018). Enunciados diretivos buscam compreender aspectos específicos que já foram mencionados pela criança. Devem ser utilizados somente se os detalhes sobre o que foi dito não foram espontaneamente esclarecidos pela criança. Não se trata de uma prática inadequada por não ser sugestiva. Em geral, envolvem questões do tipo: O quê? Quem? Quando? Como? Onde? (e.g., "Você falou que um vizinho viu o que aconteceu. Qual o nome dele?”) (Lamb et al., 2008; Lyon, 2010).

Enunciados de múltipla escolha são prejudiciais, pois questionam de maneira fechada aspectos específicos sobre eventos que não foram relatados pela criança. Tal prática pode aumentar a chance de sugerir informações e resultar em falsos relatos. Em geral, essas questões são formuladas pelas hipóteses do/a entrevistador/a (e.g., "Seu pai tocou no seu pipi?”) (Lyon, 2010). Enunciados sugestivos, além de questionar a criança sobre eventos que não foram espontaneamente relatados, indicam qual a resposta é esperada pelo/a entrevistador/a (e.g., "Seu pai tocou no seu pipi, não foi?”). Tanto as questões de múltipla escolha quanto as questões sugestivas devem ser evitadas tendo em vista o risco de contaminação dos dados coletados (Lamb et al., 2008; Lyon, 2010).

Outros aspectos relevantes a serem questionados em uma entrevista investigativa é a forma com que a revelação se estabeleceu (Lamb et al., 2008). Por vezes, trata-se de um momento em que é possível identificar pessoas protetivas para a criança e, ainda, possível revitimização causada pela não crença no relato da criança ou entrevistas repetidas (ver Santos \& Gonçalves, 2009). O encerramento da entrevista deve ocorrer de maneira semelhante ao rapport. O/A entrevistador/a deve avaliar se a criança ficou com alguma dúvida sobre a entrevista e então retomar a discussão de um tópico neutro, objetivando recuperar uma possível alteração emocional causada pelas questões investigativas (Lamb et al., 2008).

\section{Considerações Finais}

A Psicologia tem um importante papel na escuta qualificada de crianças e adolescentes vítimas de violência sexual. Conhecimentos específicos da área em relação ao desenvolvimento infantil, dinâmica da violência e procedimentos de entrevista são significativos para acolher e obter um relato suficiente que permita a proteção da vítima em diferentes órgãos da rede que compõem o Sistema de Garantia de Direitos. Profissionais dessa área podem atuar na escuta especializada ou na produção de provas em situações de suspeita de 
violência sexual contra crianças e adolescentes, seja no depoimento especial ou na perícia realizada na fase de inquérito policial ou na fase processual. $\mathrm{O}$ depoimento especial e a perícia psicológica são provas diferentes, uma sendo considerada testemunhal e a outra, pericial. A primeira envolve essencialmente a coleta de informações com a vítima ou testemunha e obteve recentemente a atenção merecida na legislação nacional (Brasil, 2017; 2018). A segunda pode incluir diferentes recursos avaliativos, é mais abrangente e só pode ser desenvolvida por profissional habilitado. Esses dois meios de prova existem para possibilitar a elucidação de situações de violência, muitas vezes permeadas de segredos e informações nebulosas. A partir da melhor elucidação, decisões mais seguras e fundamentadas são possíveis, seja para a responsabilização do/a agressor/a, seja para a proteção da vítima.

As diretrizes que caracterizam as boas práticas em entrevistas com crianças e adolescentes são fundamentais nos procedimentos de escuta especializada, depoimento especial e perícia psicológica, ainda que cada um tenha seus objetivos próprios. A literatura aborda a importância do rapport e da empatia, do encorajamento à revelação e do estabelecimento de regras iniciais de comunicação, bem como a necessidade de evitar a sugestionabilidade, entre outros aspectos fundamentais a serem considerados quando da condução de entrevistas com crianças. A qualificação de profissionais para a condução de entrevistas com crianças nesses contextos é fundamental para que se garanta a não revitimização, conforme previsto na recente legislação. Observou-se que a escuta especializada ainda requer diretrizes mais específicas para seus procedimentos, bem como a adoção de estratégias para obtenção de relato com informações mínimas para condução de posterior notificação. No entanto, ainda que careça de diretrizes específicas, as regras básicas de entrevista com crianças e adolescentes também são válidas no trabalho do profissional que atua com a escuta especializada.

O objetivo deste trabalho foi apresentar as diretrizes gerais de entrevistas com crianças e adolescentes que devem ser consideradas na atuação de psicólogos na escuta especializada, depoimento especial e perícia psicológica. Como uma revisão de literatura, este artigo restringe-se ao que está produzido e publicado em termos nacionais e internacionais. Por meio da literatura nacional, procurou-se caracterizar o contexto de trabalho e como esses três procedimentos são realizados no sistema de garantia de direitos. A partir da literatura internacional, buscou-se trazer os parâmetros esperados para condução das entrevistas forenses, que constituem as diretrizes das boas práticas que já há anos vem sendo estudadas por pesquisadores em diferentes partes do mundo. Observou-se a necessidade de qualificação dos psicólogos para a atuação nessas entrevistas, bem como a necessidade da realização de estudos nesses diferentes contextos de trabalho e procedimentos.

\section{Referências}

American Professional Society on the Abuse of Children. (2012). Practice guidelines: Investigative interviewing in cases of alleged child abuse. Chicago, IL: Author.

Aznar-Blefari, C., \& Padilha, M. G. S. (2015). Capacitação para o uso do Protocolo NICHD em profissionais sul-brasileiros. Revista de Psicología, 24(1), 1-19. doi: 10.5354/0719-0581.2015.37198

Brasil (1940). Código Penal. Brasília: Planalto Federal. Recuperado de http://www.planalto.gov.br/ccivil_03/Decreto-Lei/Del2848.htm

Brasil (1941). Código de Processo Penal. Brasília: Planalto Federal. Recuperado de http://www.planalto.gov.br/ccivil_03/decreto-lei/Del3689.htm

Brasil (1990). Lei 8.069. Estatuto da Crianca e do Adolescente. Brasilia: Presidência da República. Casa Civil. Subchefia para Assuntos Jurídicos. Recuperado de http:// www.planalto.gov.br/ccivil_03/leis/L8069.htm

Brasil (2009). Lei no 12.030, de 17 de setembro de 2009. Dispõe sobre as perícias oficiais e dá outras providências. Diário Oficial da União, Brasília (DF).

Brasil (2017). Lei 13.431. Estabelece o sistema de garantias de direitos da criança e do adolescente vítima ou testemunha de violência e altera a Lei 8069 de 13 de julho de 1990 (Estatuto da Criança e do Adolescente). Brasília: Presidência da República. Casa Civil. Subchefia para Assuntos Jurídicos. Recuperado de http://www. planalto.gov.br/ccivil_03/_ato2015-2018/2017/ lei/L13431.htm

Brasil (2018). Decreto 9.603/2018. Regulamenta a Lei $\mathrm{n}^{\circ}$ 13.431, de 4 de abril de 2017, que estabelece o sistema de garantia de direitos da criança e do adolescente vítima ou testemunha de violência. Recuperado de http://www.planalto.gov.br/ccivil_03/_Ato20152018/2018/Decreto/D9603.htm

Brown, D. A. (2011). The use of supplementary techniques in forensic interviews with children. Em M. 
E. Lamb, D. La Rooy, C. Katz, \& L. Malloy (Eds.), Children's Testimony: A Handbook of Psychological Research and Forensic Practice (2n ${ }^{\text {nd }}$ ed.), (pp. 217-249). Chichester, UK: Wiley.

Collin-Vézina, D., De La Sablonnière-Griffin, M. Palmer, A. M., \& Milne, L. (2015). A preliminar mapping of individual, relational, and social factors that impede disclosure of childhood sexual abuse. Child Abuse \& Neglect, 43, 123-134. doi: 10.1016/j. chiabu.2015.03.010

Conselho Federal de Psicologia (2009). Serviço de proteção social a crianças e adolescentes vítimas de violência, abuso e exploração sexual e suas familias: Referências para a atuação do psicólogo. Brasília: CFP

Conselho Federal de Psicologia (2018). Resolução 09/2018. Estabelece diretrizes para a realização de avaliação psicológica no exercício profissional da psicóloga e do psicólogo, regulamenta o Sistema de Avaliação de Testes Psicológicos - SATEPSI e revoga as Resoluções n. 002/2003, n. 006/2004 e n. 005/2012 e Notas Técnicas n 01/2017 e 02/2017. Brasília: Conselho Federal de Psicologia.

Conselho Nacional de Justiça (2010). Recomendação 33, de 23 de novembro de 2010. Diário da Justiça 215/2010, 33-34. Recuperado de http://www.cnj. jus.br/busca-atos-adm?documento $=1194$

Conselho Nacional do Ministério Público (2019). Guia prático para implementação da política de atendimento de crianças e adolescents vitimas ou testemunhas de violência. Conselho Nacional do Ministério Público: Brasília

Dannlowski, U., Stuhrmann, A., Beutelmann, V., Zwanzger, P., Grotegerd, D., Domschke, K., ... \& Lenzen, T. (2012). Limbic scars: Long-term consequences of childhood maltreatment revealed by functional and structural MRI. Biological Psychiatry, 71, 286-293. doi: 10.1016/j.biopsych.2011.10.021

Everson, M. D., \& Faller, K. C. (2012). Base rates, multiple indicators, and comprehensive forensic evaluations: Why sexualized behavior still counts in assessments of child sexual abuse allegations. Journal of Child Sexual Abuse, 21, 45-71. doi: 10.1080/10538712.2012.642470.

Faller, K. C. (2015). Forty years of forensic interviewing of children suspected of sexual abuse, 1974-2014: Historical benchmarks. Social Sciences, 4, 34-65. doi:10.3390/socsci4010034
Habigzang, L. F., Koller, S. H., Stroeher, F. H., Hatzenberger, R., Cunha, R. C. D., \& Ramos, M. D. S. (2008). Entrevista clínica com crianças e adolescentes vítimas de abuso sexual. Estudos de Psicologia (Natal), 13(3), 285-292. doi: 10.1590/ S1413-294X2008000300011

Habigzang, L. F., Ramos, M. S., \& Koller, S. H. (2011). A revelação de abuso sexual: As medidas adotadas pela rede de apoio. Psicologia: Teoria e Pesquisa, 27(4), 467-473. doi: 10.1590/S0102-37722011000400010

Hackbarth, C., Willams, L. C., \& Lopes N. R. L. (2015). Avaliação de capacitação para a utilização do Protocolo NICHD em duas cidades brasileiras. Revista de Psicología, 24(1), 1-18. doi: 10.5354/0719-0581.2015.36916

Herman, S. (2010). The role of corroborative evidence in child sexual abuse evaluations. Journal of Investigative Psychology and Offender Profiling, 7, 189-212. doi: 10.1002/jip.122

Hershkowitz, I., Ahern, E. C., Lamb, M. E., Blasbalg, U., Karni-Visel, Y., \& Breitman, M. (2017). Changes in interviewers' use of supportive techniques during the revised protocol training. Applied Cognitive Psychology, 31(3), 340-350. https://doi. org/10.1002/acp.3333

Hill, A. (2017). Dolls, diagrams and drawings: Interviewers' perspectives on visual aids in child witness interviews (Dissertação de mestrado). Victoria University of Wellington, Wellington.

Hohendorff, J. V., Habigzang, L. F., \& Koller, S. H. (2014). Violência sexual contra meninos: teorias e intervenção. Curitiba: Juruá.

Home Office. (2011). Achieving best evidence in criminal proceedings: Guidance for vulnerable or intimidated witnesses, including children. London, United Kingdom: Author.

Jardim, P., \& Magalhães, T. (2010). Indicadores físicos e biológicos de abuso sexual. Em T. Magalhães (Ed.), Abuso de crianças e jovens. Lisboa: Lidel.

Katz, C., Klages, A., \& Hamamma, L. (2018). Forensic interviews with children: Exploring the richness of children's drawing and the richness of their testimony. Children and Youth Services Review, 94, 557-567. doi:10.1016/j.childyouth.2018.08.034

Kendall-Tackett, K. A., Williams, L. M., \& Finkelhor, D. (1993). Impact of sexual abuse on 
children: A review and synthesis of recent empirical studies. Psychological Bulletin, 113(1), 164-180. doi: 10.1037/0033-2909.113.1.164

Lamb, M. E. (2015). Toward developmentally aware practices in the legal system: Progress, challenge, and promise. American Psychologist, 70, 686-693. doi: $10.1037 / \mathrm{a} 0039634$

Lamb, M. E., Brown, D. A., Hershkowitz, I., Orbach, Y., \& Esplin, P. W. (2018). Tell me what happened: Questioning children about abuse. Chichester: John Wiley \& Sons Ltd

Lamb, M. E., Hershkowitz, I., Orbach, Y., \& Esplin, P. W. (2008). Tell me what happened: Structured investigative interviews of child victims and witnesses. Chichester: John Wiley \& Sons Ltd

Lyon, T. D. (2010). Investigative interviewing of the child. Em D. N. Duquette, A, M, Haralambie (Eds.), Child Welfare Law and Practice (2 ${ }^{\text {nd }}$ ed.) (pp. 87-109). Denver, CO: Bradford.

Pears, K. C., Kim, H. K., \& Fischer, P. A. (2008). Psychosocial and cognitive functioning of children with specific profiles of maltreatment. Child Abuse \& Neglect, 32, 958-971. doi: 10.1016/j. chiabu.2007.12.009

Peixoto, C. E. (2011). Avaliação da credibilidade de alegações de abuso sexual de criancas: Uma perspetiva psicológica forense (Tese de doutorado). Universidade do Porto, Porto.

Peixoto, C. E., Ribeiro C., \& Alberto, I. (2013). O Protocolo de Entrevista Forense do NICHD: contributo na obtenção do testemunho da criança no contexto português. Revista do Ministério Público, 134, 181-219.

Pelisoli, C., \& Dell'Aglio, D. D. (2016). A humanização do sistema de justiça através do depoimento especial: Experiências e desafios. Psico USF, 21(2), 409-421. doi: 10.1590/1413-82712016210216

Poole, D. A., \& Bruck, M. (2012). Divining testimony? The impact of interviewing props on children's reports of touching. Developmental Review, 32(3), 165-180. doi: 10.1016/j.dr.2012.06.007

Rovinski, S. L. R., \& Pelisoli, C. L. (2019). Violência sexual contra crianças e adolescentes: Testemunho e avaliação psicológica. São Paulo: Vetor.

Salmon, K., \& Reese, E. (2016). The benefits of reminiscing with young children. Current
Directions in Psychological Science, 25, 233-238. doi: 10.1177/0963721416655100

Salmon, K., Pipe, M., Malloy, A., \& Mackay K. (2011). Do non-verbal aids increase the effectiveness of 'best practice' verbal interview techniques? An experimental study. Applied Cognitive Psychology, 26(3), 370-380. doi: 10.1002/acp.1835

Santos, B. R., \& Gonçalves, I. B. (2009). Depoimento sem medo: Culturas e práticas não revitimizantes - Uma cartografia das experiências de tomada de depoimento especial de crianças e adolescentes. São Paulo: Childhood Brasil.

Saywitz, K. J., Larson, R. P., Hobbs, S. D., \& Wells, C. R. (2015). Developing rapport with children in forensic interviews: Systematic review of experimental research. Behavioral Sciences and the Law, 33, 372-389. doi: 10.1002/bsl.2186

Schaefer, L. S. (2014). Indicadores psicológicos e comportamentais na perícia de crianças com suspeita de abuso sexual (Tese de doutorado). Pontifícia Universidade Católica do Rio Grande do Sul, Porto Alegre.

Schaefer, L. S., Brunnet, A. E., Lobo, B. O. M., Carvalho, J. C. N., \& Kristensen, C. H. (2018). Psychological and behavioral indicators in the forensic assessment of child sexual abuse. Temas em Psicologia, 26(3), 1467-1482. doi: 10.9788/TP2018.3-12En

Schaefer, L. S., Rossetto, S., \& Kristensen, C. H. (2012). Perícia psicológica no abuso sexual de crianças e adolescentes. Psicologia: Teoria e Pesquisa, 28, 227234. doi: 10.1590/S0102-37722012000200011

Whit-Woosley, A. (2019). Trauma screening and assessment outcomes in child welfare: A systematic review. Journal of Public Child Welfare, 1-23. doi: 10.1080/15548732.2019.1623965

Thierry, K. L., Lamb, M. E., Orbach, Y., \& Pipe, M. E. (2005). Developmental differences in the function and use of anatomical dolls during interviews with alleged sexual abuse victims. Journal of Consulting and Clinical Psychology, 73, 1125-1134. doi: 10.1037/0022-006X.73.6.1125

Volpini, L., Melis, M., Petralia, S., \& Rosenberg, M. D. (2016). Measuring children's suggestibility in forensic interviews. Journal of Forensic Science, 61, 104-108. doi: 10.1111/1556-4029.12987

Recebido em: 04/12/2018

Reformulado em: 27/08/2019

Aprovado em: 11/10/2019 
Sobre os autores:

Carlos Aznar-Blefari é Doutorando em Psicologia pela PUCRS, mestre em psicologia forense pela Universidade Tuiuti do Paraná.

ORCID: https://orcid.org/0000-0001-8665-5304

E-mail: psicoaznar@gmail.com

Luiziana Souto Schaefer é Doutora em Psicologia pela PUCRS, perita criminal do Instituto-Geral de Perícias do Rio Grande do Sul.

ORCID: https://orcid.org/0000-0002-7349-6532

E-mail: luiziana.schaefer@gmail.com

Cátula da Luz Pelisoli é Doutora em Psicologia pela UFRGS, psicóloga judiciária do Tribunal de Justiça do Estado do Rio Grande do Sul.

ORCID: https://orcid.org/0000-0002-7228-0449

E-mail: catulapelisoli@yahoo.com.br

Luísa Fernanda Habigzang é Doutora em Psicologia pela UFRGS, professora do Programa de Pós-Graduação em Psicologia pela PUCRS.

ORCID: https://orcid.org/0000-0002-0262-0356

E-mail: luisa.habigzang@pucrs.br

Contato com os autores:

Av. Ipiranga, 6681, Prédio 11, $9^{\circ}$ andar, sala 924

Porto Alegre-RS, Brasil

CEP: 90619-900

Psico-USF, Bragança Paulista, v. 25, n. 4, p. 625-635, out./ de\%. 2020 